\title{
Bonded Joint Elements for Structural Modeling and Failure Prediction
}

\author{
Scott E. Stapleton ${ }^{\mathrm{a}}$ and Anthony M. Waas ${ }^{\mathrm{b}}$ \\ University of Michigan, Ann Arbor, MI, 48109, USA \\ and \\ Brett A. Bednarcyk ${ }^{\mathrm{c}}$ \\ NASA Glenn Research Center, Cleveland, OH, 44135, USA
}

\begin{abstract}
Elements based on the exact stiffness matrix method contain an embedded analytical solution that can capture detailed local fields, enabling more efficient mesh independent finite element analysis. In the present study, this method was applied to adhesively bonded joints. The adherends were modeled as Euler-Bernoulli beams, and the adhesive layer was modeled as a bed of linear shear and normal springs. The field equations were derived using the principle of minimum potential energy, and the resulting solutions for the displacement fields were used to generate shape functions and a stiffness matrix for a single joint finite element. Additionally, the capability to model non-linear adhesive and adherend constitutive behavior was developed, and progressive failure of the adhesive was modeled by using a strain-based failure criteria and re-meshing the joint as the adhesive fails. Example joint configurations were analyzed to demonstrate element convergence and the modeling of functionally graded adhesives.
\end{abstract}

\section{Introduction}

$\mathrm{W}$ ith the increasing demand for composites in lightweight aerospace structures, adhesively bonded joints are becoming increasingly attractive. Bolts and rivets cause stress concentrations and premature failure in composite materials, while adhesive bonds spread the load more evenly over the composite, facilitating a lighter overall structure.

Adhesive joints have traditionally been analyzed using analytical models or finite element analysis ${ }^{1}$. Analytical methods have been utilized to extract efficient closed-form solutions for adhesive single lap joint stresses. However, analytical methods are often limited by geometric assumptions used to obtain a closed form solution and are not as useful to designers for compiling vehicle-scale models that may contain multiple joints. Finite element analyses are widely utilized in industry, and can be used to assess joints with a wide variety of geometries and loading conditions. However, these methods can suffer from mesh dependence and a lack of efficiency, which is especially crippling for initial sizing analysis and full vehicle-scale models ${ }^{2}$. Therefore, a need exists to develop predictive tools for bonded joints that can be seamlessly coupled with large scale structural analyses without

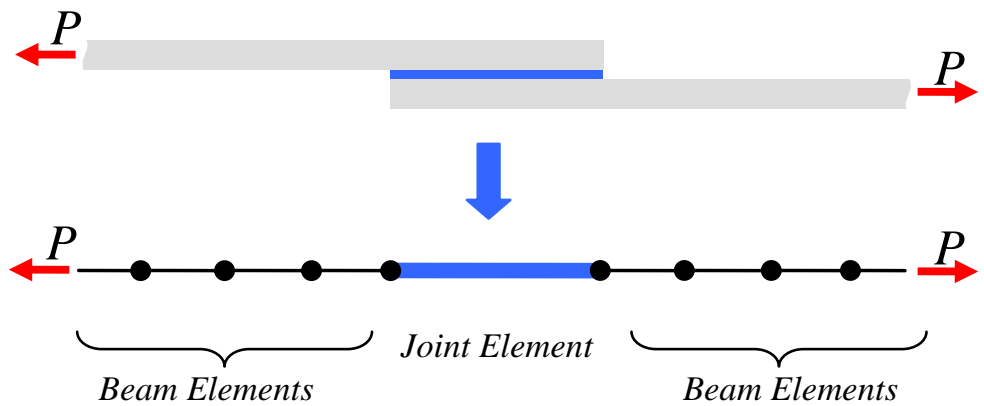

Figure 1. The present study replaces a complex single lap joint with one joint element.

${ }^{a}$ Graduate Student Research Assistant, Department of Aerospace Engineering. Email: sstaple @ umich.edu

${ }^{\mathrm{b}}$ Professor, Department of Aerospace Engineering. Associate Fellow, AIAA. Email: dcw@ umich.edu

${ }^{\mathrm{c}}$ Material Research Engineer, 21000 Brookpark Rd. Senior Member, AIAA. Email: Brett.A.Bednarcyk@NASA.gov

American Institute of Aeronautics and Astronautics 092407 
adding major computational elements. Such tools can be used to make quick mesh-independent assessments of bonded composite joints. Currently, such a capability is lacking, and joint assessment is typically performed late in the design cycle when structural changes that can lower the weight are much more difficult and expensive.

The joint element is a structural finite element made specifically for adhesively bonded joints. Motivated by the desire to create a computationally efficient tool for designing joints within a coarse, vehicle scale finite element model $^{3}$, the joint element combines an analytical formulation with a finite element. This concept has been often referred to as the exact stiffness matrix method, and has been previously applied to the beam on an elastic foundation problem. ${ }^{3,4}$ The joint element is capable of capturing the stresses in a mesh-independent, efficient manner. Such amethod is pivotal to the efficient design of composite joints, allowing the parametric studies and design optimization to take place within a larger scale finite element model. To construct the joint element, the adherends were treated as wide plates under cylindrical loading, and the adhesive was modeled as a discrete bed of normal and shear springs. The current study extends the method to include the modeling of composite adherends, functionally graded adhesives, and allows a nonlinear constitutive model for the adhesive in conjunction with inputs to cohesive zone finite element modeling, ${ }^{5,6}$ or a curve-fit for experimental test data. Additionally, a strain-based failure criterion is utilized to track damage in the adhesive, and the joint element and adjacent beam elements are resized to account for the failed adhesive. The entire joint can then be replaced by a single joint finite element, while the remaining structure (outside the joint) is modeled using standard structural elements, for instance beam elements (Fig. 1). Failure in the adhesive is then built into the joint element.

To demonstrate the usefulness of the joint element, a few examples are provided. First, a single lap joint is analyzed to show the convergence of the joint element for nonlinear adhesives. Second, the model is used to study the benefits and use of adhesives with graded properties. A baseline configuration for a single strap joint is chosen, and four different types of adhesives are compared: single modulus, step-graded (bi-adhesive), linearly graded, and exponentially graded. These functions are defined so that the grading can be described with one single variable, making optimization and parametric studies clear and simple. The effect of grading on adhesive and adherends is illustrated. Since one of the main concerns with the use of functionally graded adhesives (FGAs) is that the predesigned grading will be altered by adhesive flow during cure, a sensitivity study is conducted to show which of the functions studied is most tolerable to perturbations of the grading shape. This study demonstrates how the joint element can be used to improve the design of joints in a finite element framework.

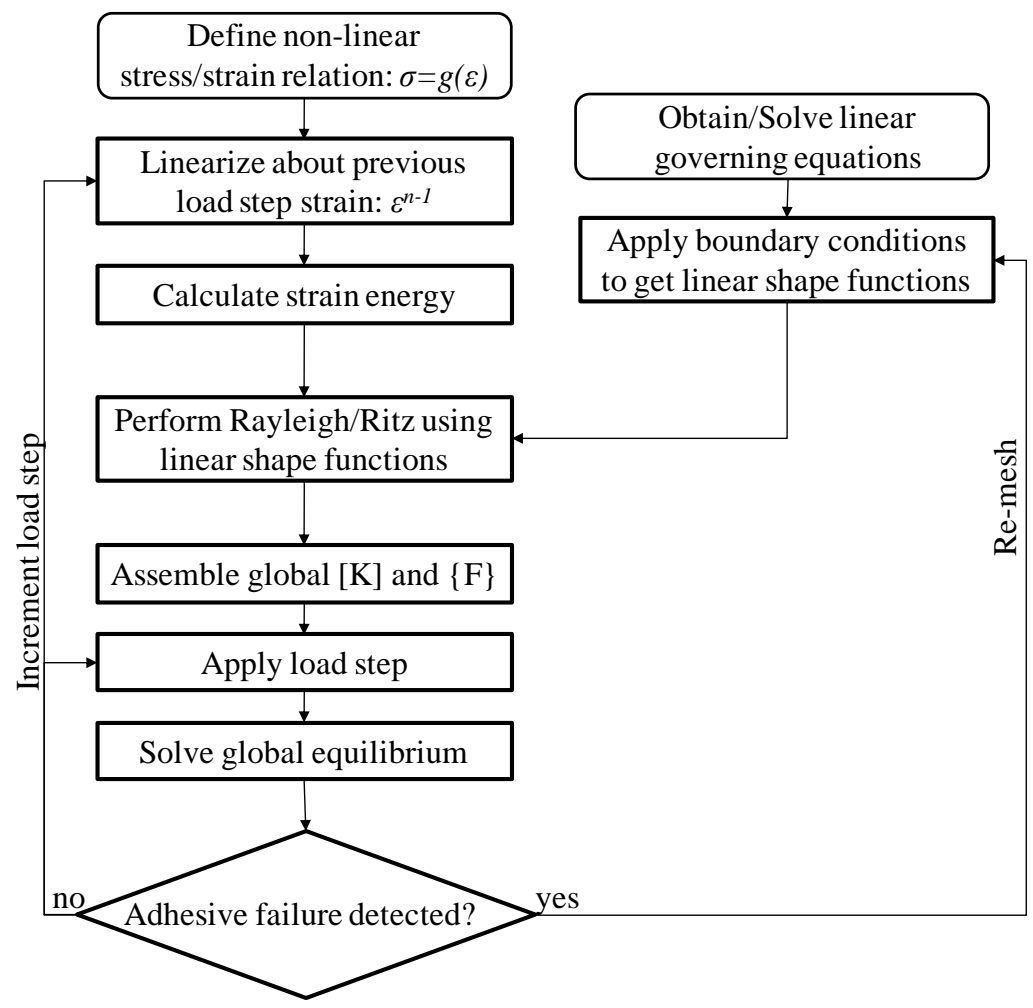

Figure 2. Flow chart of the joint element with adhesive progressive failure.

\section{Analytical Formulation}

A flow chart of the joint element implementation is shown in Fig. 2. The basic layout consists of solving the linear problem first to obtain shape functions, defining a non-linear stress/strain relation for the adhesive and linearizing this relation at each load increment, calculating the joint stiffness matrix and force vector using the linear shape functions and strain energy for the non-linear case, and incrementally solving the equations. Furthermore, the adhesive is checked for failure, and in the case of adhesive failure, re-meshing occurs to account for portions of the failed adhesive.

\section{A. Obtain Linear Shape Functions}

In order to model a nonlinear adhesive in a joint element, the shape functions are obtained from the case of a joint with a linear adhesive. This joint finite element uses an analytical formulation to get the exact stiffness 
matrix for $N$ number of adherends held together by $N-1$ adhesive layers. The adhesives and adherends were assumed to be linearly elastic, but not necessarily isotropic. The adherends were modeled as wide plates under cylindrical bending, using Euler-Bernoulli wide beam theory and Classical Lamination Theory (CLT). The adhesive response is captured through a continuous bed of shear and normal springs. This assumption ignores the traction free boundary condition which is present for an adhesive modeled as a continuum. However, most joints in application have some sort of adhesive spew coming out of the joint, which makes the imposition of a traction free boundary condition unrealistic. The material and geometric parameters are shown in Fig. 3. The subscript $i$ refers to adherend $i$, and $a_{i}$ refers to adhesive layer $i$. The width of the joint in the $y$-direction is $b$. The strain energy of the joint, $U_{\text {joint }}$, is written as:

$$
U_{j o i n t}=\sum_{i=1}^{N} \sum_{j=1}^{M_{i}} \frac{1}{2} \int_{V_{i}^{j}} \sigma_{i}^{j} \varepsilon_{i} d V+\sum_{i=1}^{N-1} \frac{1}{2} \int_{V_{a_{i}}}\left(\sigma_{a_{i}} \varepsilon_{a_{i}}+\tau_{a_{i}} \gamma_{a_{i}}\right) d V
$$

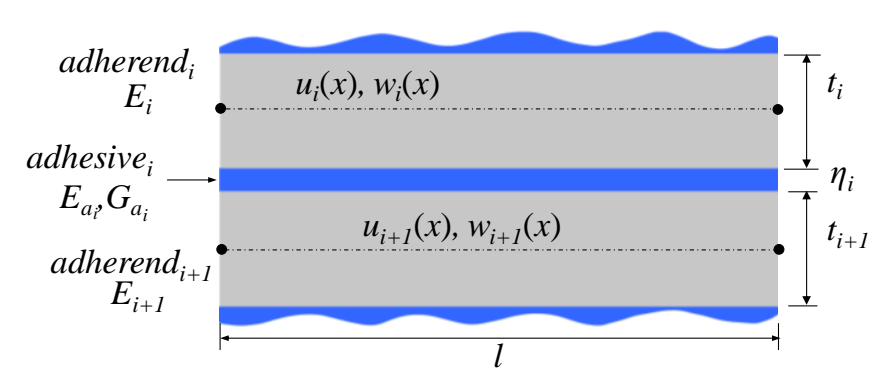

Figure 3. Geometric and material parameters for overlap region of an adhesively bonded joint.

Where $\sigma_{i}^{j}$ is the axial stress in the $j^{\text {th }}$ layer of the $i^{\text {th }}$ adherend, and $\varepsilon_{i}$ represents the axial strain in adherend $i$ in the $x$-direction. $\quad \sigma_{a_{i}}$ and $\varepsilon_{a_{i}}$ are the normal stress/strain in the $i^{\text {th }}$ adhesive in the $z$-direction, $\tau_{a_{i}}$ and $\gamma_{a_{i}}$ represent the shear stress/strain in the $i^{\text {th }}$ adhesive on the $x z$-plane, and all integrals are taken over the volume, $V_{i}^{j}$ or $V_{a_{i}}$ of adherend $i$, layer $j$ or adhesive $i$ respectively. Using CLT, and the assumptions of cylidrical bending, the stress can be written in terms of the strain and the 1,1 component of the transformed lamina stiffness matrix, $\overline{\mathbf{Q}}^{j}$ :

$$
\sigma_{i}^{j}=\bar{Q}_{11}^{j} \varepsilon_{i}
$$

and the strain can be written in terms of the adherend centerline displacements of adherend $i, u_{i}$ and $w_{i}$ :

$$
\varepsilon_{i}=u_{i, x}-z w_{i, x x}
$$

It is assumed that the displacements in the adhesive layers vary linearly in the $z$-direction and that the adhesive and adherends are perfectly bonded at the interface. The normal and shear stress in adhesive $a_{i}$ can be written in terms of the adherend displacement above and below the adhesive layer:

$$
\varepsilon_{a_{i}}=\frac{1}{\eta_{i}}\left(\left.w_{i}\right|_{z=-\frac{t_{i}}{2}}-\left.w_{i+1}\right|_{z=\frac{t_{i+1}}{2}}\right)
$$

and

$$
\gamma_{a_{i}}=\frac{1}{\eta_{i}}\left(\left.u_{i}\right|_{z=-\frac{t_{i}}{2}}-\left.u_{i+1}\right|_{z=\frac{t_{i+1}}{2}}\right)
$$

In order to be able to analyze joints with functionally graded adhesives, the Young's and shear modulus of the adhesive is kept as a general function of $x$. The stresses in the adhesive are then written as

and

$$
\sigma_{a_{i}}=E_{a_{i}}(x) \varepsilon_{a_{i}}
$$

$$
\tau_{a_{i}}=G_{a_{i}}(x) \gamma_{a_{i}} .
$$


When using bulk adhesive specimens to characterize the adhesive, the modulus must be altered to reflect a triaxial state of stess, which will be discussed in detail later.

Using the principle of stationarity of potential energy, $2 N$ fully coupled governing equilibrium differential equations are obtained from the energy expression in Eq. (1). Of the $2 N$ governing equations, $N$ equations correspond to the axial equilibrium, while $N$ equations correspond to the transverse equilibrium. The axial displacement equilibrium equations contain second order derivatives, while the transverse displacement equations have fourth order derivatives. The order of these equations can be reduced and assembled into a system of first order non-constant coefficient homogeneous ordinary differential equations of the form:

$$
\mathbf{u}_{, x}=\mathbf{A}(x) \mathbf{u}
$$

where

and

$$
\mathbf{u}=\left[\begin{array}{lllll}
\mathbf{u}_{1}^{T} & \ldots & \mathbf{u}_{i}{ }^{T} & \ldots & \mathbf{u}_{N}{ }^{T}
\end{array}\right]^{T}
$$

$$
\mathbf{u}_{i}=\left[\begin{array}{llllll}
u_{i} & u_{i, x} & w_{i} & w_{i, x} & w_{i, x x} & w_{i, x x x}
\end{array}\right]^{T} .
$$

In order to solve the system of equations found in Eq. (6), a semi-numerical method of solution was adopted. Traditional differential equation solving techniques employing numerical boundary conditions could not be employed because the boundaries (nodes) contain unknown, symbolic conditions. Therefore, the domain was broken up into segments in which the coefficient matrix, $\mathbf{A}(x)$, is considered constant and solved using the matrix exponential. First, consider segment $n+1$, with a local $x$-direction coordinate system $x$ ' which originates at the left side of the segment, $x=x_{n}$. The other end of the segment is at $x^{\prime}=\Delta x$ and $x=x_{n+1}$.

It is assumed that $\Delta x$ is significantly small so that $\mathbf{A}(x)$, can be considered constant within each such segment. The linearized coefficient matrix $\mathbf{A}_{n+1}$, is taken to be the coefficient matrix evaluated at the midpoint of the segment:

$$
\mathbf{A}_{n+1}=\mathbf{A}\left(x_{n}+\frac{\Delta x}{2}\right)
$$

Within segment $n+1$, the system can now be expressed as a system of ordinary constant coefficient differential equations in the local coordinate system, $x$, of the form:

$$
\mathbf{u}_{, x^{\prime}}=\mathbf{A}_{n+1} \mathbf{u} .
$$

Inspecting the matrix $\mathbf{A}_{n+1}$ can be helpful in determining the nature of the solution and determining the solution method. There are $6 N$ eigenvalues of $\mathbf{A}_{n+1}: N$ real eigenvalues, $2 N$ complex eigenvalues, and $3 N$ repeating eigenvalues. Therefore, the solution is made up of $N$ exponential terms, $2 N$ exponential terms multiplied by a sine or cosine, and the $3 N$ repeating eigenvalues correspond to a third order polynomial found in a standard beam solution. Such a complex solution shows that merely employing standard beam shape functions to the joint problem would be inadequate in capturing the nature of the complete solution.

The solution of the system in Eq. (8) can be written in terms of the matrix exponential, $\mathbf{e}^{\mathbf{A}_{n+1} x^{\prime}}$, and a vector of unknown constants, $\mathbf{C}_{n+1}$, as

$$
\mathbf{u}\left(x^{\prime}\right)=\mathbf{e}^{\mathbf{A}_{n+1} x^{\prime}} \mathbf{C}_{n+1} .
$$

The matrix exponential can be expressed as the infinite series ${ }^{7}$

$$
\mathbf{e}^{\mathbf{A}_{n+1} x^{x^{\prime}}}=\sum_{k=0}^{\infty} \frac{x^{k}}{k !} \mathbf{A}^{k}
$$


In order to get faster convergence, a method of scaling and squaring ${ }^{8}$ is employed, and the series is calculated up to a value of $k$ which yields an acceptable error, $\varepsilon$. The error can be defined many ways, but the current study defined the error as the difference between the 1-norms of $\mathbf{e}^{\mathbf{A}_{n+1} x^{\prime}}$ for $k-1$ and $k$. The value of the acceptable error was set at $\varepsilon=0.0001$. In the local coordinate system, the solution $\mathbf{u}_{n}$ at $x{ }^{\prime}=0$ can be expressed as

$$
\mathbf{u}_{n}=\mathbf{e}^{\mathbf{A}_{n+1} 0} \mathbf{C}_{n+1}=\mathbf{C}_{n+1},
$$

and the solution, $\mathbf{u}_{n+1}$, at the end of the segment $\left(x^{\prime}=\Delta x\right)$ can be written in terms of the solution at the beginning of the segment,

$$
\mathbf{u}_{n+1}=\mathbf{e}^{\mathbf{A}_{n+1} \Delta x} \mathbf{u}_{n}
$$

to eliminate the vector of constants, $\mathbf{C}_{n+1}$. Similarly, the solution at the end of the previous segment can be expressed as

$$
\mathbf{u}_{n}=\mathbf{e}^{\mathbf{A}_{n} \Delta x} \mathbf{u}_{n-1}
$$

and so on, down to the first segment, which has the solution:

$$
\mathbf{u}_{0}=\mathbf{e}^{\mathbf{A}_{0} \Delta x} \mathbf{C}_{0}
$$

Therefore, the solution at any segment, $n+1$, can be expressed in terms of the vector of constants from the first segment, $\mathbf{C}_{0}$, by the equation:

$$
\mathbf{u}_{n+1}=\mathbf{e}^{\mathbf{A}(x) x_{n+1}} \mathbf{C}_{0}
$$

where

$$
\mathbf{e}^{\mathbf{A}(x) x_{n+1}}=\prod_{m=0}^{n+1} \mathbf{e}^{\mathbf{A}_{m} \Delta x} .
$$



Figure 4. Boundary conditions for adherend i: prescribed nodal displacements and rotations at $x=0$ and $x=l$.
The next step is to solve for the vector of constants, $\mathbf{C}_{0}$, using the boundary conditions. This is where the analytical formulation is discretized and the displacements are obtained in terms of the nodal displacements as defined in Fig. 4. For adherend $i$, the boundary conditions on the left side of the joint $(x=0)$ can be expressed in the following equation:

$$
\mathbf{q}_{i 0}=\mathbf{b}_{c} \mathbf{u}_{i}(0)
$$

where $\mathbf{u}_{\mathrm{i}}(0)$ is $\mathbf{u}_{\mathrm{i}}$ evaluated at $x=0, \mathbf{q}_{i 0}$ is a vector containing the prescribed nodal degrees of freedom of adherend $i$ at $x=0$, and

$$
\mathbf{b}_{c}=\left[\begin{array}{llllll}
1 & 0 & 0 & 0 & 0 & 0 \\
0 & 0 & 1 & 0 & 0 & 0 \\
0 & 0 & 0 & 1 & 0 & 0
\end{array}\right]
$$

Eq. (18) for all $N$ adherends can be assembled together, and a relation between the nodal degrees of freedom at $x=0$ and the vector of constants can be found using Equation (16): 
where

$$
\mathbf{q}_{0}=\mathbf{B}_{N} \mathbf{e}^{\mathbf{A}(x) 0} \mathbf{C}_{0}
$$

$\mathbf{q}_{0}=\left[\begin{array}{lllll}\mathbf{q}_{10}{ }^{T} & \ldots & \mathbf{q}_{i 0}{ }^{T} & \ldots & \mathbf{q}_{N 0}{ }^{T}\end{array}\right]^{T}$

and

$$
\mathbf{B}_{N}=\left[\begin{array}{lll}
\mathbf{b}_{c} & & \\
& \ddots & \\
& & \mathbf{b}_{c}
\end{array}\right]
$$

where the subscript $N$ denotes the number of matrices on the diagonal. After performing the same operations at $x=l$, all of the boundary conditions can be gathered together in the form

$$
\mathbf{z C}_{0}=\left\{\begin{array}{c}
\mathbf{q}_{0} \\
\mathbf{q}_{l}
\end{array}\right\}=\mathbf{q}
$$

where

$$
\mathbf{Z}=\mathbf{B}_{2 N}\left\{\begin{array}{l}
\mathbf{e}^{\mathbf{A}(x) 0} \\
\mathbf{e}^{\mathbf{A}(x) l}
\end{array}\right\} .
$$

Using this relation, one can obtain an expression for the vector of unknown constants:

$$
\mathbf{C}_{0}=\mathbf{z}^{-1} \mathbf{q}
$$

This relation can be inserted into Eq. (16) to get the adherend centerline displacements in terms of the nodal degrees of freedom,

$$
\mathbf{u}=\mathbf{N q},
$$

where the exact shape functions, $\mathbf{N}$ are defined as:

$$
\mathbf{N}=\mathbf{e}^{\mathbf{A}(x) x} \mathbf{z}^{-1}
$$

\section{B. Define Adhesive Stress/Strain Relation}

With the shape functions determined for a joint with a linear adhesive, the nonlinear constitutive stress/strain relations of the adhesive need to be defined. This can be based on measured stress-strain relations, or inferred stress-strain relations from fracture properties. A stress/strain relationship based on a measured tensile stress/strain curve can be fitted with a fitting function. An inferred stress-strain relation that uses fracture properties would use a function which has its maximum stress at the Mode I critical stress $\left(\sigma_{I c}\right)$ and the area under the curve would correspond to the critical strain energy release rate of the adhesive $\left(G_{I C}\right)$. Regardless of the method used to define the relation, the adhesive stress, $\sigma_{a z}$, can be written as a function of the adhesive strain, $\varepsilon_{a z}$ :

$$
\sigma_{a z}=g\left(\varepsilon_{a z}\right)
$$


Although this paper refers to the normal stress and strain in the adhesive only, the same derivation holds for the shear strain/stress relation. Similarly, a nonlinear relation can be used for the adherends, which is particularly appropriate for many metals which display a significant amount of ductility before fracture. It should also be noted that this stress/strain relation assumes no permanent plasticity but resembles nonlinear elasticity. Since the failing adhesive domain is eliminated in the iteration process (to be described later), the assumption of a nonlinear elastic type stress-strain law suffices for this modeling process since regions of "unloading" are minimal.

Often, the function from Eq. (28) is defined based on bulk adhesive experimental data. However, the tensile loading of

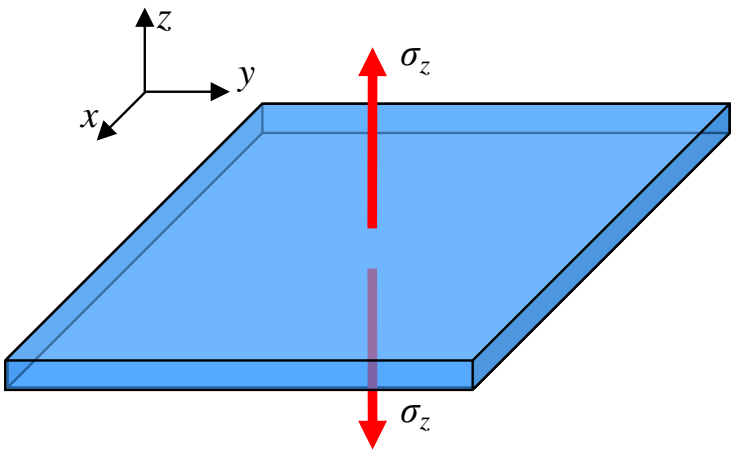

Figure 5. A thin adhesive layer with an applied stress is in a state of triaxial stress.

a thin adhesive layer with relatively large in-plane dimensions differs greatly to that of a bulk adhesive specimen because the adhesive layer is extremely thin in one direction, and constrained from lateral displacement by the top and bottom adherends. Because of these conditions, the adhesive is effectively a body in plane strain in the two directions perpendicular to the adhesive thickness (Fig. 5). The adhesive is constrained from contracting (Poisson's effect) in the $\mathrm{x}$ and $\mathrm{y}$-directions while being loaded in the $\mathrm{z}$-direction, which induces a stress in all three directions, commonly called a state of triaxial stress ${ }^{9}$. To find the stress/strain relation for a material under triaxial stress, consider first an isotropic, linearly elastic material. The normal stress in the z-direction is:

$$
\sigma_{a z}=\frac{E_{a}}{1+v_{a}}\left(\varepsilon_{a z}+\frac{v_{a}}{1-2 v_{a}}\left(\varepsilon_{a z}+\varepsilon_{a x}+\varepsilon_{a y}\right)\right) .
$$

The adhesive can be assumed to be in a state of plane stress in the $x y$-plane, and the strains $\varepsilon_{a x}$ and $\varepsilon_{a y}$ can be set to zero. Then, the normal stress in the $z$-direction reduces to:

$$
\sigma_{a z}=\frac{1-v_{a}}{\left(1+v_{a}\right)\left(1-2 v_{a}\right)} E_{a} \varepsilon_{a z}
$$

This shows that the effective "resistance" to deformation in the $z$-direction is amplified by a factor that depends on Poisson's ratio. Although this relation is intended for linear elasticity, the relation was assumed to hold for the nonlinear stress/strain relation as well. Therefore, the stress/strain relation was redefined as:

$$
\sigma_{a z}=\frac{1-v_{a}}{\left(1+v_{a}\right)\left(1-2 v_{a}\right)} g\left(\varepsilon_{a z}\right)
$$

which effectively increases the adhesive modulus.

\section{Linearize Stress/Strain Relation}

To simplify calculations and avoid the need for a nonlinear solver, the loading is broken up into increments and the stress/strain relation of the adhesive is linearized about the previous strain increment. The Taylor series expansion of the stress at the $(n+1)^{\text {th }}$ increment, can be written in terms of the strain at load step $n, \varepsilon^{n} \quad$ as:

$$
\sigma\left(\varepsilon^{n+1}\right)=g\left(\varepsilon^{n}\right)+\frac{d g\left(\varepsilon^{n}\right)}{d \varepsilon} \Delta \varepsilon+H O T
$$

where HOT represents higher order terms, $\Delta \varepsilon=\varepsilon^{n+1}-\varepsilon^{n}$, and the subscript $a z$ has been dropped from the stress and strain symbols. To linearize, the higher order terms are ignored. 


\section{Calculate the Adhesive Strain Energy}

The adhesive normal strain energy $U^{n+1}$ at the next load step, $n+1$, is found as the strain energy from the previous increment plus the integral of the stress as a function of strain from the previous increments to the current increment:

$$
U^{n+1}=\int_{V_{a}} \int_{\varepsilon^{n}}^{\varepsilon^{n+1}} \sigma(\varepsilon) d \varepsilon d V+U^{n} .
$$

Carrying out the inner integral gives:

$$
U^{n+1}=\int_{V_{a}}\left(\frac{1}{2} \frac{d g\left(\varepsilon^{n}\right)}{d \varepsilon}\left(\varepsilon^{n+1}\right)^{2}+\left(g\left(\varepsilon^{n}\right)-\frac{d g\left(\varepsilon^{n}\right)}{d \varepsilon} \varepsilon^{n}\right) \varepsilon^{n+1}-g\left(\varepsilon^{n}\right) \varepsilon^{n}-\frac{1}{2} \frac{d g\left(\varepsilon^{n}\right)}{d \varepsilon}\left(\varepsilon^{n}\right)^{2}\right) d V+U^{n} .
$$

\section{E. Perform Rayleigh/Ritz Using Linear Adhesive Shape Functions}

To obtain the stiffness and force matrices for the joint, the shape functions derived for the linear adhesive case (Eq. 5) are used. Using Eq. (2) and (4) and the shape functions derived for the linear adhesive case (Eq. (22)), the strain in the adhesive is found in terms of the nodal displacements, $q_{1-12}$. The strain in the adhesive at the current, $n$ +1 , increment is written as a function of $x$ and $q_{1-12}$ :

$$
\varepsilon^{n+1}=f\left(q_{1-12}\right)
$$

while the displacements from the previous increment are used to define the adhesive strain at the previous increment, $\varepsilon^{n}$, as a function of $x$ only. The energy is then minimized, which yields the $i^{\text {th }}, j^{\text {th }}$ component, $k_{\mathrm{ij}}$, of the contribution to local joint stiffness matrix from the adhesive normal strain:

$$
k_{i, j}=\frac{\partial^{2}}{\partial q_{i} \partial q_{j}} \int_{v_{a}} \frac{1}{2} \frac{d g\left(\varepsilon^{n}\right)}{d \varepsilon}\left(\varepsilon^{n+1}\right)^{2} d V
$$

and the $i^{\text {th }}$ component contribution of the adhesive normal strain to the local joint force vector, $f_{i}$, to be

$$
f_{i}=\frac{\partial}{\partial q_{i}} \int_{v_{a}}\left(\frac{d g\left(\varepsilon^{n}\right)}{d \varepsilon} \varepsilon^{n}-g\left(\varepsilon^{n}\right)\right) \varepsilon^{n+1} d V .
$$

Using the same steps, a similar relation can be derived for the adhesive shear or the adherend normal components. The contribution of the adhesive normal and shear strains to the local force vector and stiffness matrix can be added to the contributions of the two adherends. The local stiffness matrix and local force vector can be used to find the local nodal displacements, $\mathbf{q}$ :

$$
\mathbf{k q}=\mathbf{f} .
$$

\section{F. Assemble Global Matrices, Apply Loading, and Solve Global Equations}

Once the joint element stiffness matrix and load vector are found, they are assembled with the rest of the elements in the model. The loading increment is applied, and the system of linear equations is solved at each increment. For this particular study, an in-house finite element code was used to assemble and solve the finite element global equations. 




Figure 6. The building block approach facilitates modeling complex joints with simple joint element building blocks.
This formulation gives the exact stiffness matrix for a simple region of constant thickness adherend overlap. However, many joints in application contain complicated geometries, including ply steps and tapers. To use the simple joint element for efficient modeling of complex joints, a building block approach was implemented. This approach involves combining simple, constant-thickness joint sections to create complicated joints with very few elements. This

concept is illustrated in Fig. 6, where single, double and triple adherend joint building blocks are combined to make complicated joints such as a PI joint, tapered single lap joint, and spliced sandwich joint. Adherends joined together at the adherend centerlines are related to each other with the equation

$$
q_{1 L}=q_{1 R}, i=1 . .3
$$

where the subscript $L$ is for the adherend on the left, $R$ is for the adherend on the right, and the numerical subscripts 1,2 , and 3 refer to axial, transverse, and rotational degrees of freedom respectively. To model a ply step or taper, the transverse and rotational degrees of freedom are equal, but the axial degree of freedom of the left adherend is related to that of the right adherend through the following equation:

$$
q_{1 L}=q_{1 R}-t_{o f f s e t} q_{3 R}
$$

where $t_{\text {offset }}$ is the vertical ( $z$-direction) offset distance between the two nodes.

\section{G. Checking for Adhesive Failure}

Once the nodal displacements are found, Eq. (2a) and (2b) are used to find the strain in the adhesive as a function of horizontal position, $x$. Then, a strain-based failure criterion based on the failure strain, $\varepsilon_{f a i l}$, is used to determine if and how much the adhesive has failed (Fig. 7a).

If failure is detected, the joint element is shortened by the length of the failed adhesive region, and the adjacent beam elements are lengthened to compensate (Fig. 7b). After this step is completed, the steps described in are repeated until the joint reaches equilibrium. After equilibrium is reached and further failure no longer occurs, the load is increased by one increment, and the process is repeated. Through this method, the stresses, strains, loads, and displacements for the joint can be found at each load increment as the joint deforms nonlinearly and fails progressively. 


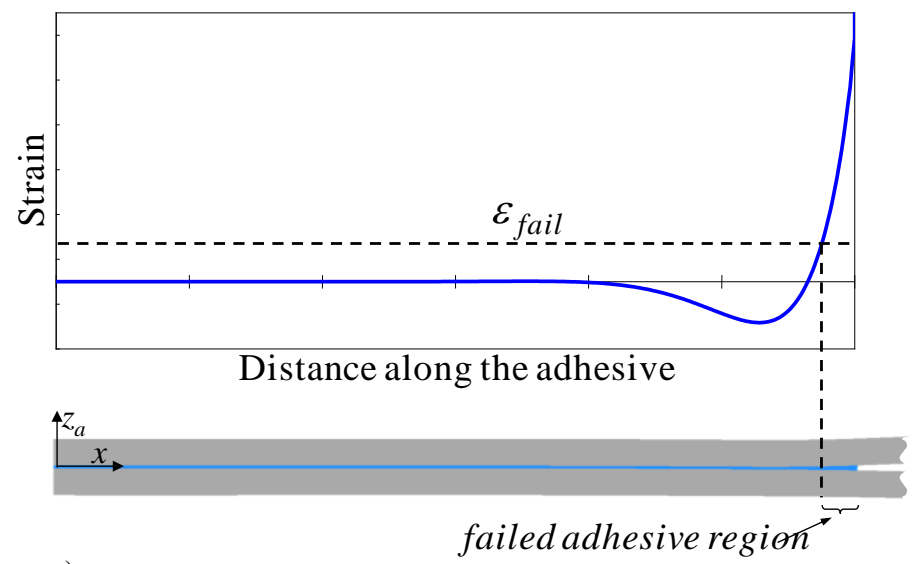

a)

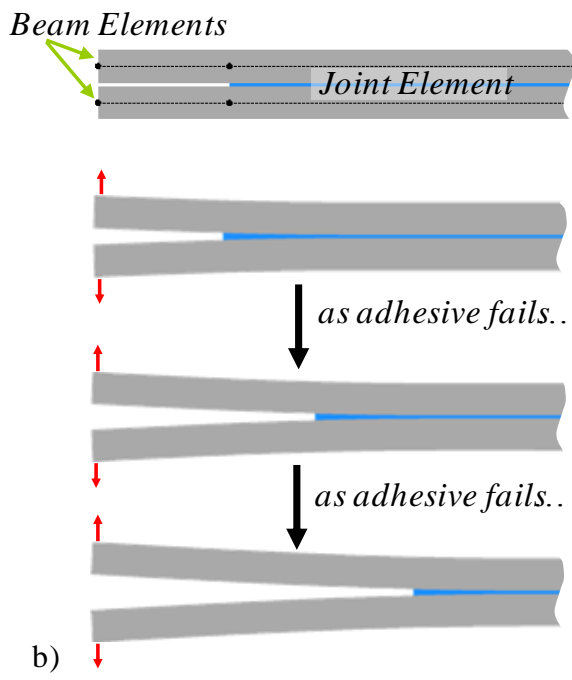

Figure 7. Once a region of adhesive exceeds the a) failure strain, this region is considered failed and $b$ ) the joint element is shortened while the adjacent beam elements are lengthened (DCB geometry depicted).

\section{Results}

\section{A. Single Lap Joint}

An example configuration of a single lap joint was modeled with the joint element to show convergence. Convergence can be an issue because the shape functions used for the joint element came from the linearly elastic adhesive case. Once significant softening of the adhesive occurs, the shape functions are no longer exact for the problem. Since this method was created to allow a joint to be modeled with a single element, it is important to determine how much of an effect using the linearly elastic adhesive shape functions has on the stress and failure of a joint.

The configuration along with the loading and boundary conditions are found in Fig. 8a. The joint overlap was modeled using one element, eight elements, and 32 elements in the overlap region (Fig. 8b). The adherends were made of aluminum, and the material and geometric parameters are found in Table 1. The stress/strain behavior of the adhesive was based on the bulk adhesive tensile data of ESP105 epoxy as reported by Harris and Adams ${ }^{10}$ (Fig. 8c). The tensile response was modeled by fitting a tanh function to the aforementioned data. This function was chosen because it resembled the form of the response and it gives the same response for compression and tension. The shear response was not obtained experimentally, but rather assumed based on the tensile response. First, the Poisson's ratio was taken to be 0.34 , and the shear modulus was obtained based on the initial slope of the tensile curve and Hooke's law. The maximum stress of the shear response was found by assuming that the adhesive behaves according to $\mathbf{J} 2$ flow theory, and that this holds true after initial yield. The shear failure strain was calculated by assuming that the strain energy at failure was the same for shear and tensile loading. The shear and tensile responses of the adhesive were kept uncoupled, and adhesive failure was initiated when either the shear or normal strain reached the corresponding failure value.

Table 1. Material properties and geometric parameters for the single lap joint example ${ }^{10}$.

\begin{tabular}{ccccccc}
\hline \multicolumn{2}{c}{ Adherend } & \multicolumn{4}{c}{ Geometric Parameters } \\
$\boldsymbol{E}(\mathbf{G P a})$ & $\boldsymbol{v}$ & $\boldsymbol{l}(\mathbf{m m})$ & $\boldsymbol{L}(\mathbf{m m})$ & $\boldsymbol{b}(\mathbf{m m})$ & $\boldsymbol{t}(\mathbf{m m})$ & $\boldsymbol{\eta}(\mathbf{m m})$ \\
\hline 70 & 0.33 & 12.7 & 63.5 & 24.1 & 1.6 & 0.125 \\
\hline
\end{tabular}


a)

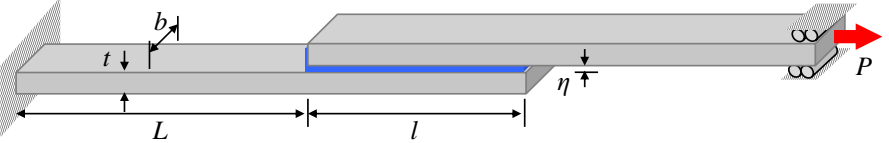

b)

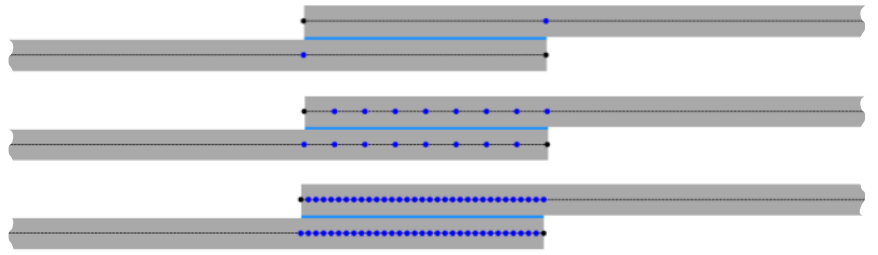



c)

Strain, $\mathbf{m m} / \mathbf{m m}$

Figure 8. Single lap joint: a) geometric parameters, b) joint element representations with one, eight, and 32 joint elements across the overlap region, and c) curve fit of bulk tensile stress/strain response for ESP105 adhesive ${ }^{10}$ along with an approximated shear response.

The difference in joint strength predictions between models with different numbers of joint elements is illustrated in Fig. 9. Using one element still gives a reasonable answer, and more than two elements are needed to get within $5 \%$ of the converged strength. The shear and peel stress in the adhesive layer at a load of $5.2 \mathrm{kN}$ for one,

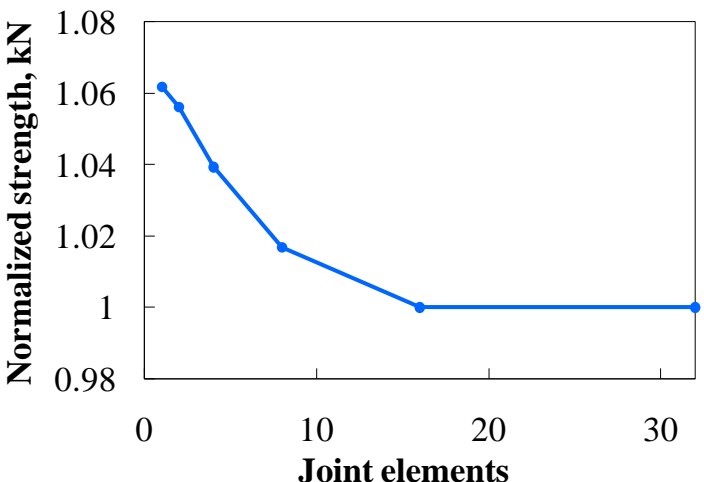

Figure 9. Convergence of joint strength for the single lap joint with increasing joint elements.

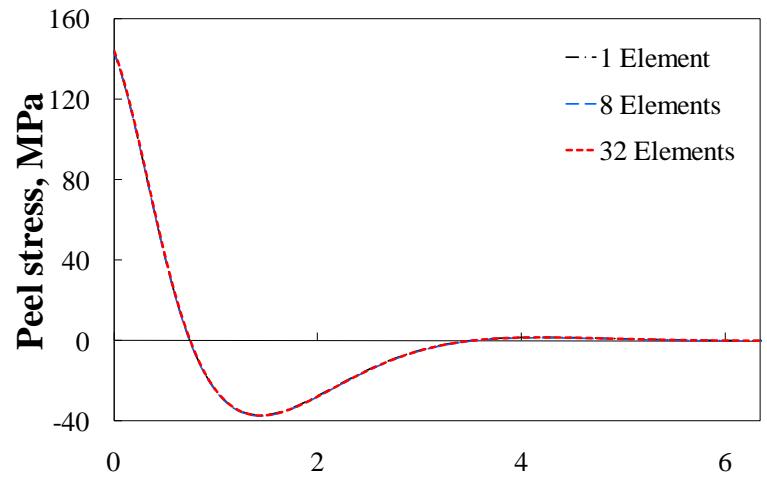

a)

Distance, $x$, along adhesive, $\mathrm{mm}$ eight, and 32 elements is shown in Fig. 10. The peel stresses are almost identical for all cases, mainly due to the fact that less softening has occurred in the $z$ direction. There is a larger difference in shear stress between the models with one, eight, and 32 elements because the adhesive has softened more in shear. This descrepancy can be explained by looking at the tangential modulus shown in Fig. 11. The adhesive has softened significantly at the ends, making the tangential modulus a function of $x$. The shape functions were found for a constant modulus adhesive, which no longer represents the softened adhesive. Therefore, the shape functions do not represent the exact solution after softening has occurred. To improve the solution, the shape functions would have to be calculated at each load increment based on the tangential modulus at the previous increment. In this way, the joint could be modeled with one element without a loss in accuracy.

Figure 10. Convergence study for the joint element modeling a single lap joint with ESP105 adhesive: a) adhesive peel stress and b) adhesive shear stress using one, eight, and 32 elements to model the joint overlap. 
A major source of error for this type of joint is the fact that large displacements and rotations were not taken into account in the joint element formulation. The joint already had rotations in excess of $1^{\circ}$ at $13 \%$ of the maximum load, making this problem highly nonlinear with respect to geometry. Harris and Adams ${ }^{10}$ reported this joint to have a strength of $9.9 \pm 0.65 \mathrm{kN}$, while the joint element model predicted a joint strength of $5.8 \mathrm{kN}$. This illustrates the need to include large rotations when modeling single lap joints, making it imperative that geometric nonlinearities be included to model the strength of single lap joints. This capability will be included in future versions of the model.

As a side note, it has been observed that adhesive softening is responsible for the spreading of the stress in the joint in a more even manner. ${ }^{10,11}$ The adhesive properties become naturally graded along the joint to minimize the



Figure 11. Tangent shear and normal modulus of single lap joint prior to failure. stress concentration (Fig. 11). The same effect can be achieved by artificially grading the adhesive along the length of the adhesive. Such a grading can decrease the maximum stress of the joint without incurring any damage of the adhesive.

\section{B. Single Strap Joint with Functionally Graded Adhesive}

In order to demonstrate the usefulness of the joint element, functionally graded adhesives (FGAs) are studied. A single strap joint (or butt end joint) was chosen as the baseline configuration because there is a single dominant stress concentration in the middle of the joint, which lends itself to single variable optimization and parametric studies. The geometric and material properties are defined in Fig. 12a, and the finite element representation is shown in Fig. 12b. Half of the joint was modeled due to symmetry, and the overlap section was modeled by one or several joint elements (depending on the number of discrete regions of continuous adhesive modulus) while the nonjoint adherend section is modeled with one beam element. The loaded end is constrained from rotation and vertical translation, while the symmetric face of the doubler is constrained from horizontal translation and rotation. The values of the material and geometric parameters used for the analytical modeling are found in Table 2. The FGAs were compared with two different single adhesive systems Fig. 13a. These two adhesives provided upper and lower bounds for the grading functions, and will be referred to as $E_{u}$ and $E_{l}$ respectively. The grading functions chosen for investigation included a step (bi-adhesive), linear, and exponential function. These functions were all reduced to one single grading variable, $l$, as defined in Fig. $13 \mathrm{~b}$, c, and d. For the step function, $l$ is the length of the more compliant adhesive. For the linear function adhesive, $l$ is the length of the section in which the modulus decays linearly, and for the exponential function it is the length of adhesive which has a modulus less than $99 \%$ of $E_{u}$. Although these may not be the optimal grading functions, these functions were chosen because of their ability to be reduced to one variable, allowing for simplistic and clear sensitivity studies. It was assumed that no matter what the grading, the relationship between the Young's modulus and shear modulus remained constant, or in other words that the Poisson's ratio remained constant. A similar assumption was made by Apetre et al. ${ }^{12}$ for functionally graded sandwich beam cores.

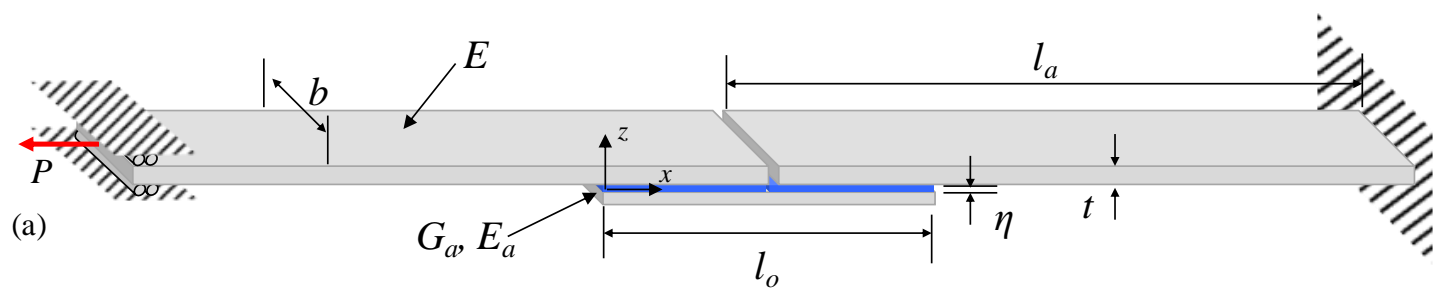

(b)

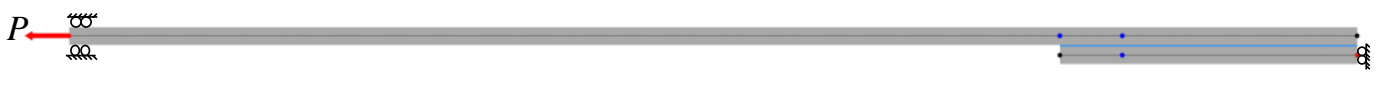

Figure 12. Single strap joint (a) geometric and material parameters and (b) joint finite element representation assuming symmetry. 
Table 1: Parameters of the baseline single strap joint configuration used for the theoretical study.

\begin{tabular}{ccccccccccc}
\hline $\begin{array}{c}\boldsymbol{P} \\
(\mathbf{k N})\end{array}$ & $\begin{array}{c}\boldsymbol{l}_{\boldsymbol{a}} \\
(\mathbf{m m})\end{array}$ & $\begin{array}{c}\boldsymbol{l}_{\boldsymbol{o}} \\
(\mathbf{m m})\end{array}$ & $\begin{array}{c}\boldsymbol{t} \\
(\mathbf{m m})\end{array}$ & $\begin{array}{c}\boldsymbol{b} \\
(\mathbf{m m})\end{array}$ & $\begin{array}{c}\boldsymbol{\eta} \\
(\mathbf{m m})\end{array}$ & $\begin{array}{c}\boldsymbol{E} \\
(\mathbf{G P a})\end{array}$ & $\begin{array}{c}\boldsymbol{E}_{\boldsymbol{u}} \\
(\mathbf{G P a})\end{array}$ & $\begin{array}{c}\boldsymbol{E}_{\boldsymbol{l}} \\
(\mathbf{G P a})\end{array}$ & $\boldsymbol{v}$ & $\begin{array}{c}\boldsymbol{\tau}_{\text {net }} \\
(\mathbf{M P a})\end{array}$ \\
\hline 4000 & 82.6 & 38.1 & 1.1 & 25.4 & 0.4 & 108.5 & 2.5 & 1.1 & 0.34 & 8.3 \\
\hline
\end{tabular}

Most stress values reported in the theoretical study were normalized by the net shear stress, $\tau_{\text {net }}$, defined by:

$$
\tau_{n e t}=\frac{P}{l_{o} b}
$$

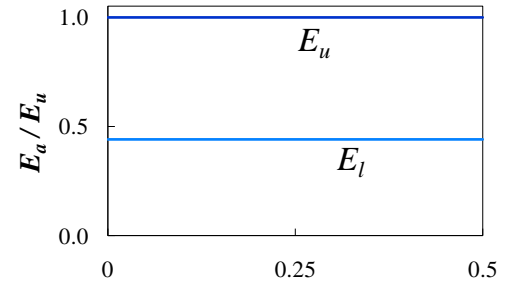

a)
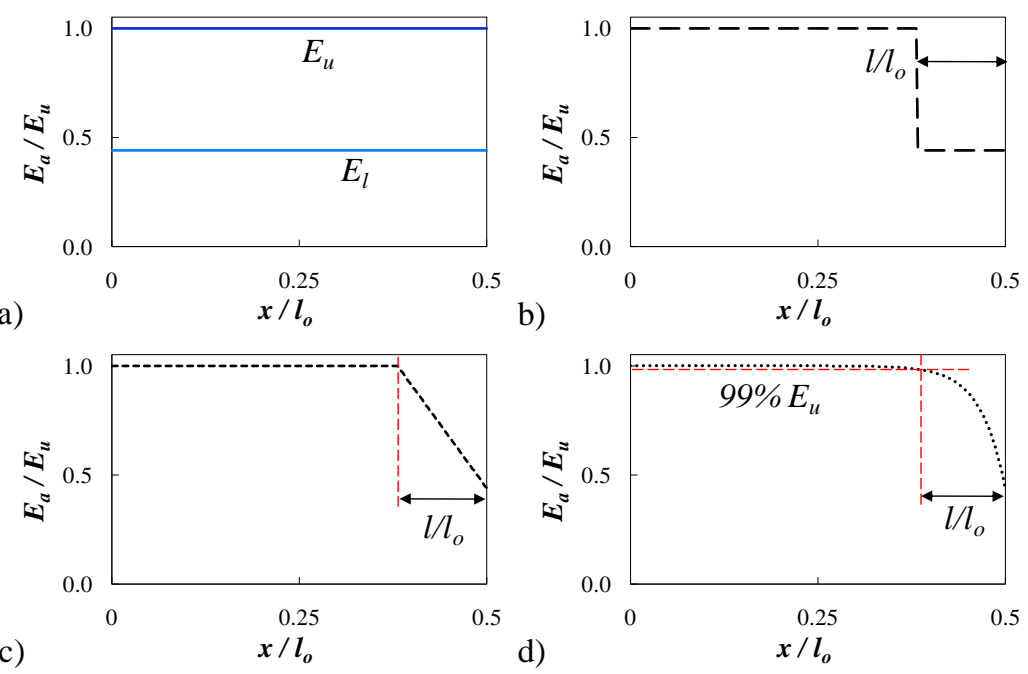

Figure 13. Single strap joints with (a) constant modulus adhesives were compared with joints with functionally graded adhesives, including (b) step-wise graded, (c) linearly graded, and (d) exponentially graded.

To show the benefits of using FGAs, the grading parameter, $l$, was optimized to reduce the maximum peel stress in the adhesive for the three FGAs, and the resulting moduli are plotted in Fig. 15a. As shown, the region of gradation is very small, about $2 \%$ of the overlap length for the step and linear function adhesives, and around 5\% for the exponential. The peel stress in the adhesive for half of the symmetric joint is plotted in Fig. $15 \mathrm{~b}$ for each adhesive. The single adhesive joints are in blue, and the FGAs are in black. The step FGA has two stress peaks; one at the end of the adhesive and one at the interface between the two adhesives. The linear and exponential FGAs have a rounded stress peak, and appear to result in very similar stress distributions.

The maximum stress in the adherend, doubler, and adhesive for the single adhesive joints and the optimized FGA joints is found in Fig. 16. All stress values are normalized by the maximum stress found in the stiffer single adhesive joint in order to plot all stresses in the same plot. The stress reported for the adherend and the doubler is the normal stress in the $\mathrm{x}$-direction, and the maximum value of the stress is found at the upper and lower surfaces of the adherend and doubler respectively. With composite laminate adherends, the most important stress in the adherend is usually the peel stress ( $z$-direction) between the plies because failure often initiates there. However, the current configuration contains only one ply, so this stress cannot be captured.

Some important aspects of using FGAs are illustrated in Fig. 9. First, the FGAs in this study outperformed the stiffer single adhesive joint, $E_{l}$. Adhesive stresses were considerably lower and adherend and doubler stresses were not significantly impacted. This is important because the more compliant single adhesive joint had lower adhesive stresses, but higher adherend stress. The FGAs were able to lower the adhesive stress without affecting the adherend stress. Second, when compared with the $E_{l}$ adhesive, the FGAs reduced the adhesive peel stress but did not necessarily reduce the shear stress. It will be shown later that the optimum value of the grading variable $l$ is not the same for minimizing peel as it is for minimizing shear stress. Therefore, the relative levels of peel and shear must be considered when designing FGAs so that the dominant stress can be minimized. However, typical adhesives are more ductile under shear loading, so peel stresses will normally be the minimized variable. 


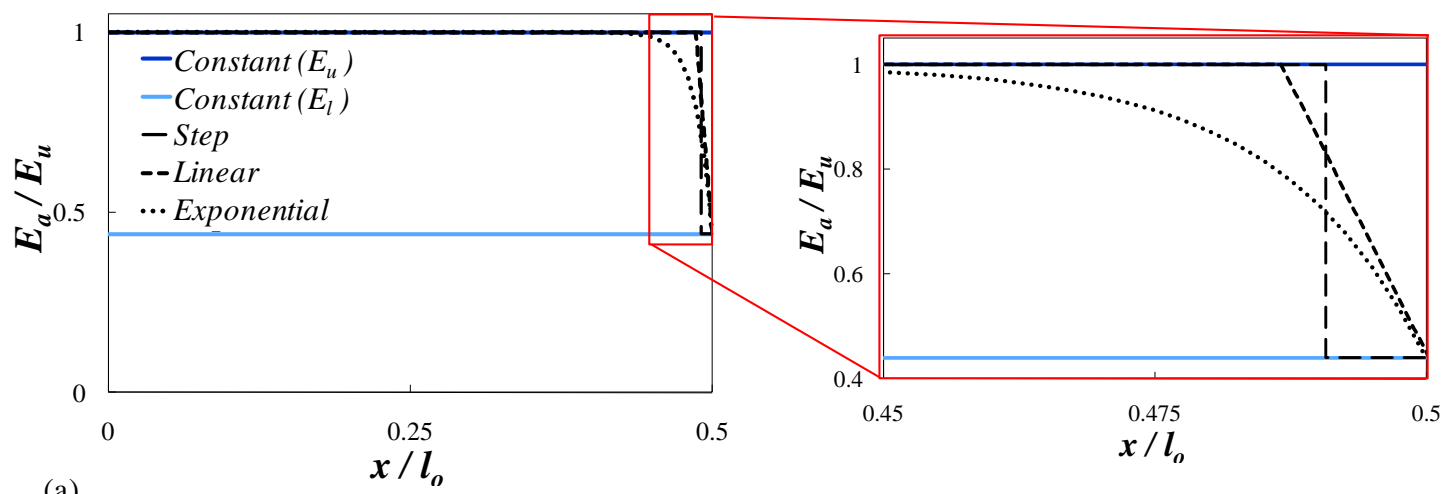

(a)

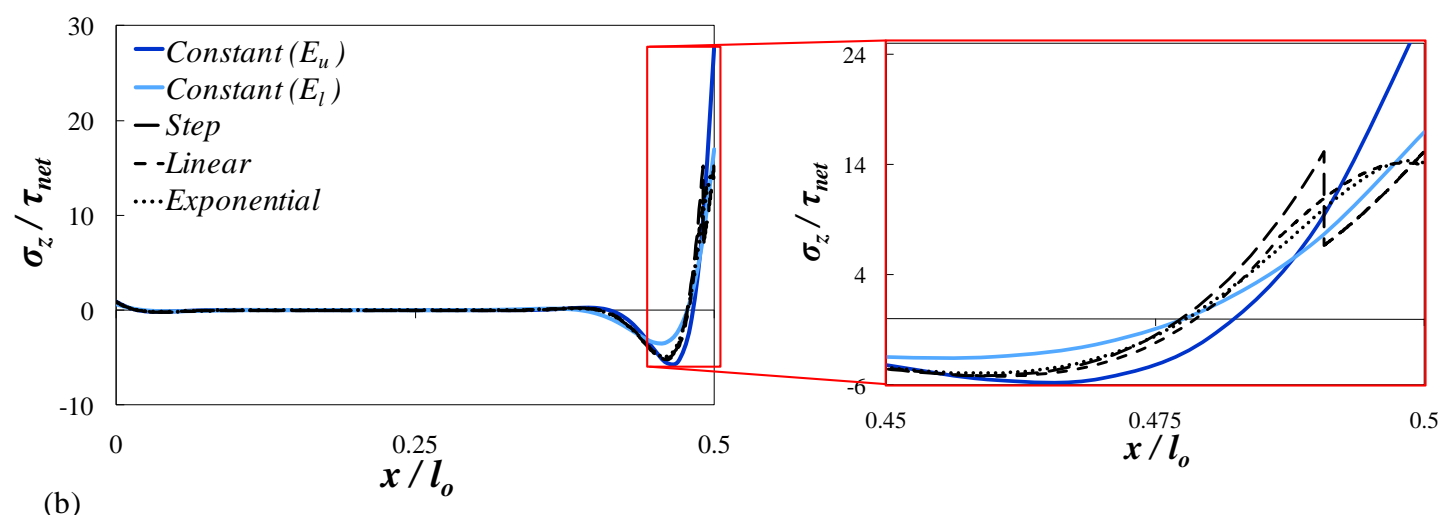

Figure 14. Optimized configurations for the single strap joints for different functions of graded adhesive compared: (a) modulus across the adhesive and (b) centerline peel stress across the adhesive.

Another aim of this study was to address the concern that during manufacturing, the adhesive is pressurized and heated, often causing the adhesive to flow and even squeeze out of the joint. If a functionally graded adhesive (FGA) is specifically designed for a certain joint, this could either change the shape of the grading, the lower bound modulus, or both. This could result in an FGA which has higher stress than using the more compliant adhesive alone. This section seeks to address this concern by presenting a grading sensitivity study. For the purposes of this study, sensitivity will refer to the narrowness of the range of $l$ values which results in lower maximum stresses than those obtained using the lower bound adhesive, $E_{l}$. The effects of changing the grading parameter $l$ on the maximum adhesive stress are quantified Fig. 16. Through this study, insight is gained into how sensitive the grading is and which functions are more tolerant to changes in the grading.

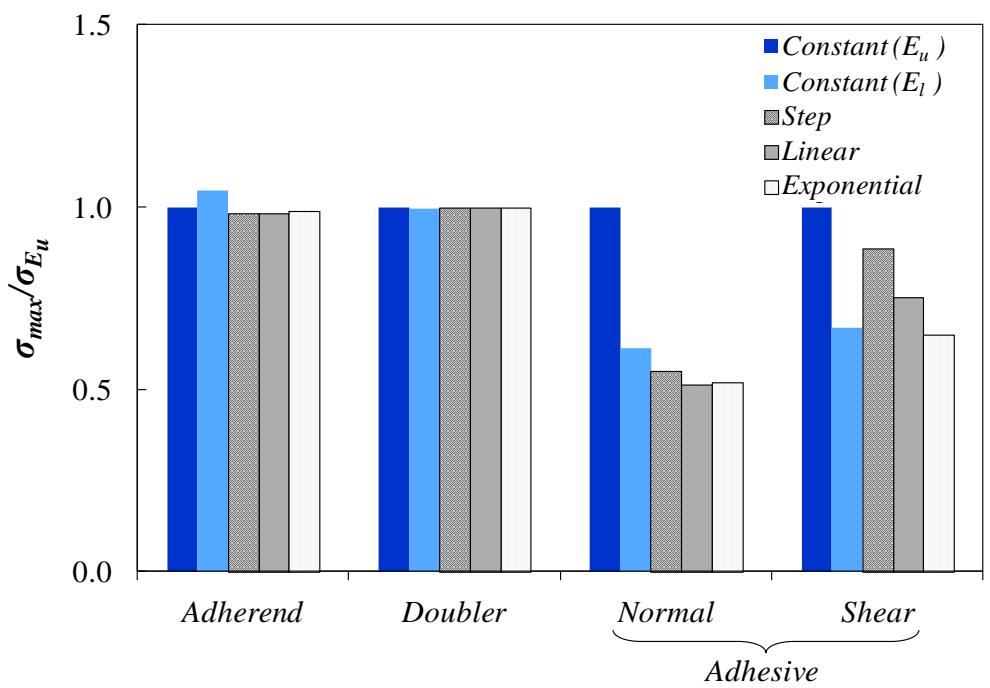

Figure 15. Comparison of the maximum stresses in joints with different adhesives, where all maximum stress values are normalized by the maximum stress value found in the stiffer single adhesive joint. 


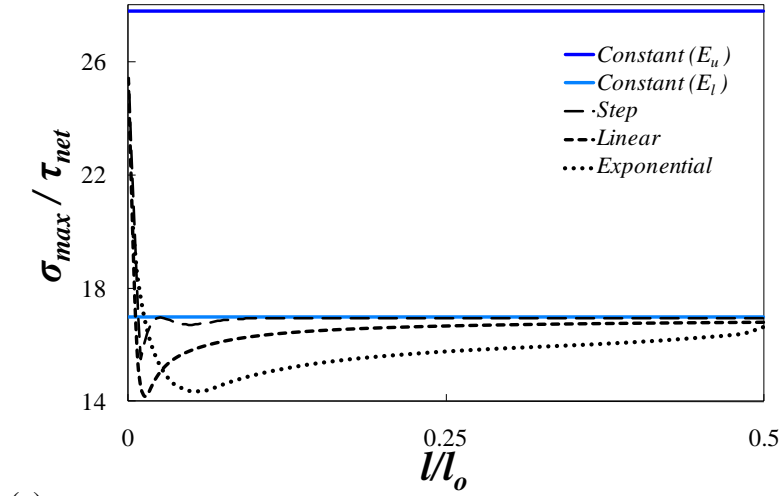

(a)

Figure 16. Observing the maximum adhesive (a) peel and (b) shear stress as a function of the grading parameter $l$ shows how sensitive the maximum stress is to the shape of the grading.

The effects of changing the grading parameter $l$ on the maximum adhesive peel and shear stresses for the three FGAs are found in Fig. 16a and 16b respectively. For all three FGAs, the maximum shear stress is less sensitive to the grading parameter than the peel stress. Also, the optimum value of $l$ is always greater for minimizing the shear stress than the peel stress. This is most likely because the peel stress peak is much more concentrated than the shear stress peak, so a steeper gradation is needed to minimize the peak. Also, if $l$ becomes too short, the stress goes above the stress which would be found in a joint with just the more compliant adhesive. Unfortunately, with the addition of pressure, a decreasing $l$ is more likely. The step function adhesive was the most sensitive: only a very small range of values of $l$ results in lower stresses than just using the more compliant adhesive, $E_{l}$. The linear function adhesive was not as sensitive, and it converges to a stress less than that of $E_{l}$ when $l$ is large. Finally, the exponential function adhesive had a broad range of $l$ values resulting in low maximum stress, making it the most tolerant to changing the grading parameter.

\section{Conclusions}

Bonded joint elements that use the exact stiffness matrix method, based on an analytical solution, have been used to study two types of adhesively bonded joints. Such elements can approximately capture the behavior of an entire joint by using an analytical method to solve for the appropriate shape functions rather than prescribe the shape functions using a polynomial interpolation. This joint element is intended for use as a design tool which can model a joint in a mesh-independent manner and still couple with global vehicle-scale finite element models. As a design tool, it is not intended to replace high-fidelity detailed models, but enable fast, efficient sizing and design of bonded joints.

Progressive failure was included in the model by defining the stress as a nonlinear function of the strain and enforcing an uncoupled strain-based failure criterion. Progressive failure of the adhesive was approximated by shortening the joint element by the length of the failed adhesive and lengthening the adjoining beam elements. The shape functions obtained for a joint with a linearly elastic adhesive were utilized for the nonlinear adhesive case. Softening of the adhesive as the stress increases causes the shape functions to no longer be exact. However, a mesh convergence study conducted for a single lap joint configuration showed that the difference in strength predictions between using one joint element and 32 were only approximately $6 \%$. The predicted strength for a single lap joint did not match well with an experimental result from the literature, most likely because of a lack of large rotation inclusion in the joint element, which will be added in future versions of the element. Additionally, it was shown how softening of the adhesive layer where the stress is the highest causes the adhesive to spread the stress more evenly across the joint. Such an effect could be replicated without adhesive softening by artificially grading the modulus of the adhesive across the joint.

Bonded joints with functionally graded adhesives (FGAs) were also studied to show the usefulness of the joint element in parametric studies and joint design. A single strap joint with four joint systems (single adhesive, biadhesive, linear, and exponential functionally graded adhesives) were analyzed. It was shown that for the single strap joint configuration investigated, FGAs could reduce the maximum peel stress in the adhesive by up to $17 \%$ over the more compliant single adhesive joint without having an adverse effect on the adherend stress. Since the optimum grading for shear stress was not the same as that for peel stress, there was a slight increase in shear stress 
when peel stress was minimized. However, all grading functions resulted in a significant reduction (> 45\%) in peel stress over the stiffer single adhesive joint without adversely affecting the load carrying capability or the stress in the adherends.

The study also addressed a practical concern about using FGAs, which is that the flow of adhesive during manufacturing would change the shape of the grading and cause the grading to be ineffective. Therefore, a sensitivity study was conducted on the three FGAs to see the effect of changing the shape of the grading $(l)$ to reflect what might occur when adhesive is squeezed out of the joint. The exponential FGA proved to be quite tolerant to changes in grading shape. This could be the basis for a justification for using the more complicated exponential FGA over the bi-adhesive. Although the peel stress reductions were all very similar, the tolerability of the exponential grading to perturbation of grading shape, which can occur due to adhesive flow, might make it worth the extra complications.

As was demonstrated in the study, the joint element can be a very useful tool for efficiently exploring a wide variety of joint concepts early on in the design phase. Additionally, such studies can be part of a global-scale structural FE model where the design of the joint can be coupled with the overall design of the entire vehicle.

\section{Acknowledgements}

This work was financially supported in part by the Space Vehicle Technology Institute under grant NCC3-989 jointly funded by NASA and the Department of Defense. It was managed within the NASA Constellation University Institutes Project, with Claudia Meyer as the project manager and H. Kevin Rivers \& Stanley Smeltzer (NASA Langley), as the project monitors. Additional financial support was provided by NASA Glenn Research Center through the GSRP Fellowship and the authors are grateful for the advice and support of Dr. Steven Arnold of the NASA Glenn Research Center

\section{References}

${ }^{1}$ Gustafson, P. A. and Waas, T. A. "A Macroscopic Finite Element for a Symmetric Double-lap Joint Subjected to Mechanical and Thermal Loading," International Journal for Numerical Methods in Engineering, Vol. 79, 2009 , pp. 94-126.

2 Zhang, J, Bednarcyk, B. A., Collier, C, Yarrington, P., Bansal, Y., and Pindera, M. J., "Analysis Tools for Adhesively

Bonded Composite Joints Part 2: Unified Analytical Theory,” AIAA Journal, Vol. 44, No. 8, 2006, pp. 1709-1719.

${ }^{3}$ Eisenberger, M. and Yankelevsky, D. Z., "Exact Stiffness Matrix for Beams on Elastic Foundation," Computers and Structures. Vol. 21, 1985, pp. 1355-1359.

${ }^{4}$ Aydogan, M., "Stiffness-Matrix Formulation of Beams with Shear Effects on Elastic Foundation," Journal of Structural Engineering, Vol. 121, 1995 pp. 1265-1270.

5 ABAQUS, Inc, ABAQUS user Manuel v6.7-1, Electronic Version, 2006.

6 Tsai, M. Y. and Morton, J., "An evaluation of Analytical and Numerical Solutions to the Single-Lap Joint," Int. J. Solids Structures, Vol. 31, No. 18, 1994, pp. 2537-2563.

7 Chen, C., Linear System Theory and Design, $3^{\text {rd }}$ ed., Oxford University Press, New York, 1999.

8 Molder, C. and Van Loan, C., "Nineteen Dubious Ways to Compute the Exponential of a Matrix, Twenty-Five Years Later", SIAM Review, Vol. 45, No. 1, pp 3-49.

9 Sechler, E., Elasticity in Engineering, Wiley: Chapman \& Hall, New York, 1952.

${ }^{10}$ Harris, J. A., and Adams, R. D., "Strength prediction of bonded single lap joints by non-linear finite element methods," International Journal of Adhesion and Adhesives, Vol. 4, 1984, pp. 65-78.

${ }^{11}$ Mortensen, F. and Thomsen, O.T., "Analysis of Adhesive Bonded Joints: a Unified Approach," Composites Science and Technology, Vol. 62, 2002, pp. 1011-1031.

12 Apetre, N. A, Sankar, B. V., Ambur, D. R., "Low-velocity impact response of sandwich beams with functionally graded core," International Journal of Solids and Structures, Vol. 43, 2006, pp. 2479-2496. 